\title{
La necesaria renovación de la formación del profesorado para una educación científica de calidad
}

\author{
Amparo Vilches* \\ Daniel Gil Pérez*
}

Artículo recibido: 2-8-2007 y aprobado: 12-10-2007

Resumen: Se analiza en primer lugar, a la luz de la investigación, cuáles han sido los progresos realizados en el campo de la formación del profesorado de ciencias durante las dos últimas décadas así como las dificultades encontradas.

Tomando en consideración los resultados de dicho análisis, se intentan fundamentar propuestas de renovación de la formación del profesorado para el logro de una educación científica de calidad que responda a las nuevas necesidades sociales.

Palabras clave: formación del profesorado de ciencias, alfabetización científica, inmersión en la cultura científica, aprendizaje como investigación.
Articulo recibido: 2-8-2007 y aprobado: 12-10-2007

Abstract: This study initially analyses, in the light of research, the progress made in the field of science teacher training over the last two decades, as well as the difficulties encountered.

Bearing in mind the results of the analysis described above, this paper aims to make proposals to renew teacher training in order to achieve a quality science education that meets the new needs of society.

Key words: Science teacher training; basic science education; immersion in science culture; learning as research.

1 Este artículo ha sido concebido como contribución a la Década de la Educación para un futuro sostenible, instituida por Naciones Unidas para el periodo 2005-2014. (Ver http://www.oei.es/decada/).

* Universitat de Valencia

Amparo.Vilches@uv.es; http://www.uv.es/vilches/

daniel.gil@uv.es; http://www.uv.es/gil/ 


\section{Introducción: dos décadas de avances... insuficientes}

Hace 20 años, en 1987, la National Association for Research in Science Teaching de Washington D.C. organizó un simposio para establecer, a la luz de la investigación educativa, los conocimientos y destrezas que necesitan poseer los profesores de ciencias (Hewson y Hewson, 1988). Cabe destacar que, aunque la preocupación por la formación del profesorado como uno de los factores esenciales del proceso enseñanza/ aprendizaje es antigua, los estudios solían centrarse en las características del buen profesor (Ausubel, 1978), mientras que en dicho simposio la cuestión se planteó en términos de cuáles son los conocimientos que los profesores necesitamos adquirir. El matiz fue sin duda importante, porque suponía el cuestionamiento de concepciones esencialistas ("se es", "se nace" buen profesor) que apuntaban a ineficaces políticas de selección más que a procesos de formación (Gil Pérez, 1991). Una formación que pasó a ser considerada imprescindible para hacer frente a los graves problemas que planteaba la educación científica: fracaso escolar y desinterés e incluso rechazo por los estudios científicos.

Desde entonces la preocupación por la educación científica y, muy en particular, por la formación del profesorado no ha hecho más que crecer (Windschitl, 2005). Así, en un reciente estudio promovido por la Comisión Europea (Rocard et ál., 2007) se reconoce que numerosas investigaciones han mostrado en estos últimos años un alarmante descenso del interés de los jóvenes por los estudios científicos. Algo que constituye, según se afirma en el informe Rocard, una de las mayores amenazas para el futuro de Europa, dada la necesidad tanto de un número creciente de científicos y tecnólogos, como de una alfabetización científica básica de toda la ciudadanía para hacer posible su participación en la toma fundamentada de decisiones. Y se añade que el origen de este descenso del interés está, en buena medida, en la forma en que la Ciencia es enseñada, lo que dirige la atención hacia la formación del profesorado, que constituye la piedra angular de cualquier renovación de la educación científica.

Consideraciones similares acerca de la necesidad de renovar la enseñanza y la formación del profesorado, las encontramos en el ámbito iberoamericano (Maiztegui et ál., 2000). Así, por ejemplo, en la Declaración de la XV Conferencia Iberoamericana de Ministras y Ministros de Educación, que tuvo lugar en Toledo en $2005^{2}$, podemos leer: "las reformas educativas de la última década derivan en un conjunto importante de demandas respecto de los contenidos, las modalidades y el alcance de la formación inicial y continua del profesorado que tengan un impacto directo en las prácticas pedagógicas del aula".

Cabe señalar que esta creciente preocupación por la renovación de la educación científica y la formación del profesorado no supone que todo continúe igual o peor que hace dos décadas. En realidad las propuestas actuales se apoyan en un avance fundamental: los responsables de los diseños curriculares han asumido mayoritariamente los resultados de la investigación educativa

\footnotetext{
2 http://www.oei.es/xvcie.htm
} 
en lo que respecta a las estrategias de trabajo en el aula susceptibles de favorecer actitudes de interés hacia las ciencias y un mejor aprendizaje. La necesidad de sustituir las estrategias de transmisiónrecepción por otras que orienten el aprendizaje como una tarea de indagación o investigación, que favorezca la participación de los estudiantes en la re-construcción de los conocimientos, concita hoy un consenso general entre los expertos. Ésa es la tesis básica del informe encargado por la Comisión Europea (Rocard et ál., 2007): sabemos que es necesario introducir en los centros orientaciones basadas en la indagación (Inquiry-based Science Education, IBSE), desplazando el tradicional chalk and talk; la investigación y los numerosos ejemplos de experiencias controladas con éxito así lo prueban (Abd-El-Khalick et ál., 2004, Rudolph, 2005; Trumbull et ál., 2005; Lee et ál., 2006).

Tesis similares fueron también, ya hace más de diez años, el punto de partida de los National Science Education Standards en EEUU (National Academy of Sciences, 1995), un documento de fácil acceso $^{3}$ que merece ser estudiado y cuyo capítulo 4 está dedicado al desarrollo profesional de los profesores para hacer posible la introducción en el aula de las nuevas estrategias de aprendizaje como investigación.

En definitiva, hay consenso acerca de qué estrategias promover en el aula. El problema estriba, como reconoce el Informe Rocard (2007), en que el profesorado se muestra, en general, reticente a los cambios propuestos.

\footnotetext{
3 http://www.nap.edu/readingroom/books/nses/
}

Resulta necesario, pues, analizar con algún detenimiento, a la luz de lo que la investigación ha ido mostrando, las causas de estas resistencias, y sobre todo, averiguar qué estrategias formativas pueden resultar adecuadas para que los educadores cumplamos con la doble tarea de estimular el interés por la cultura científica del conjunto de la ciudadanía y de preparar adecuadamente a un número suficiente de candidatos para proseguir estudios científico-tecnológicos superiores. Una doble necesidad que aparece asociada al creciente reconocimiento de que la Educación ha dejado de ser solamente una inversión estratégica "a futuro", para convertirse en un factor esencial de desarrollo, también a corto plazo (National Academy of Science, 1995; Fourez, 1997).

Éste es el propósito de nuestra contribución con la que intentaremos mostrar, fundamentadamente, es decir, tomando en consideración las investigaciones e innovaciones realizadas por la comunidad internacional -recopiladas en Handbooks, manuales y proyectos (Anderson y Mitchener, 1994; National Academy of Science, 1995; Mumby y Russell, 1998; Perales y Cañal, 2000; Gil Pérez, Macedo et ál., 2005)-, que los problemas actuales de la educación científica tienen solución. Más aún, que estamos en un momento de auténtica crisis, en su sentido más positivo de "mutación importante en el desarrollo". Pero que ello exige el cuestionamiento de un cierto número de concepciones (ideas, actitudes y comportamientos), asumidas acríticamente -actitud muy frecuente en el campo educativo y, en particular, en lo que atañe a la formación y actividad de los docentes (Anderson 
y Mitchener, 1994; Gil Pérez y Vilches, 2004)- que se convierten por ello en auténticos obstáculos para la renovación de la educación científica.

\section{Hacia la superación de planteamientos reduccionistas de la formación del profesorado}

La necesidad de asociar las reformas educativas a una correcta formación del profesorado aparece, según hemos visto, como la primera lección a extraer de las dificultades aparecidas en los procesos de reforma curricular. Pero es preciso reconocer que ello constituye un lugar común que, aparentemente, ha sido siempre tenido en cuenta. Así, como mostraron Anderson y Mitchener (1994) en su revisión de la investigación acerca de la formación del profesorado de ciencias -publicada en el Handbook of Research on Science Teaching and Learning (Gabel, 1994)-, los promotores de las reformas educativas señalan sistemáticamente a la formación del profesorado como el punto de partida necesario para que sean posibles los cambios; pero, lamentablemente, ello no se suele traducir en estudios en profundidad acerca de cuáles deben ser las orientaciones de dicha formación.

No basta, pues, con afirmar la importancia de la formación del profesorado en los procesos de reforma. Para muchos, ello significa simplemente insistir en la preparación habitual de los contenidos científicos, añadiendo algunos elementos genéricos de Educación desligados de los contenidos. Esta separación de los contenidos científicos y educativos se ha mostrado, sin embargo, muy poco eficaz. Como señala McDermott (1990), el uso efectivo de una estrategia de en- señanza viene, a menudo, determinado por el contenido. Si los métodos de enseñanza no son estudiados en el contexto en el que han de ser implementados, los profesores pueden no saber identificar los aspectos esenciales ni adaptar las estrategias de enseñanza -que les han sido presentadas en términos abstractos- a su materia específica o a nuevas situaciones. McDermott concluye, en consecuencia, con un rechazo de esta suma de formación científica y preparación docente independientes entre sí. Una crítica semejante ha sido realizada por numerosos autores (Maiztegui et ál., 2000). Sin embargo, es este tipo de formación el que, a menudo, se ha propuesto y sigue proponiéndose en numerosos países a los profesores activos, como preparación para hacer posibles los cambios curriculares.

Se hace necesario, pues, salir al paso de ésta y otras orientaciones que han mostrado ya sus limitaciones, pero que siguen presentes en propuestas bien intencionadas como la del ya mencionado Informe Rocard (2007). En efecto, la principal dificultad que el informe contempla para que el profesorado pase a utilizar las estrategias de aprendizaje como investigación es la falta de un buen conocimiento de la materia: muchos maestros de primaria -se señala- carecen de suficientes conocimientos para enseñar ciencias, por lo que recurren a la simple transmisión, con la cual se sienten más cómodos, y evitan las estrategias de indagación, que requiere una comprensión más profunda.

Es cierto que las estrategias de indagación requieren (y hacen posible) una comprensión más profunda de la materia enseñada, por lo que su falta 
se convierte en un serio obstáculo. Pero se trata de un análisis escorado porque las estrategias de chalk and talk no afectan exclusivamente a los maestros de primaria. En realidad afectan muy particularmente al profesorado universitario, cuyos conocimientos no son puestos en duda.

Aquí encontramos una primera y grave limitación en éste y en la mayoría de los estudios acerca de la formación del profesorado: la universidad es dejada absolutamente de lado cuando se habla renovar la enseñanza para hacer posible el interés de los jóvenes hacia la Ciencia. Es cierto que los jóvenes que se desinteresan o incluso rechazan los estudios científicos sólo están en contacto con profesores de primaria y secundaria pero, ¿acaso la forma como enseñan sus profesores no tiene nada que ver con la forma como se enseña en la universidad? Este reduccionismo a la hora de focalizar el problema -cuestionando exclusivamente la enseñanza primaria y secundaria- supone una grave limitación, insistimos, porque las formas de enseñar en los distintos niveles se potencian mutuamente: lo que viven en la universidad los futuros profesores supone una impregnación muy efectiva, tanto por su duración cuanto por tener lugar en una etapa de plena madurez intelectual.

De hecho, los National Standards for Science Education (National Academy of Sciences, 2005) insisten en que la educación científica, en todos los niveles, debe basarse en la metodología de la investigación, como forma de favorecer, tanto una actividad significativa en torno a problemas susceptibles de interesar a los estudiantes, como su progresiva autonomía de juicio y capacidad de participación en tareas colectivas. Y, por supuesto, es en el nivel universitario donde esta metodología de investigación es potencialmente más efectiva (Black, 2000; Martínez Torregrosa, Gil Pérez y Martínez Sebastián, 2003). Cabe señalar que estas estrategias formativas son las utilizadas habitualmente en la universidad con aquellos postgraduados que se incorporan a algún departamento para prepararse como futuros investigadores. Y cabe señalar también que este periodo es vivido, por la generalidad de quienes pasan por él, como el más fructífero de su formación. Sin embargo, dicha concepción está totalmente ausente de la realidad de las aulas universitarias del primer y segundo ciclo. La omnipresente transmisión de conocimientos ya elaborados, las colecciones de ejercicios resueltos como "no problemas", y las "prácticas de laboratorio" desarrolladas a modo de recetas y desligadas de la estructura lógica de las asignaturas, parecen insustituibles, por diversas razones en las que no podemos entrar aquí, pero entre las que puede jugar un papel importante, pensamos, la separación que la universidad suele establecer entre investigación y carga docente.

Cabe esperar que esta situación de la enseñanza universitaria se modifique para hacer frente a los problemas de la propia universidad, pero, en cualquier caso, el cambio es urgente en lo que se refiere a la formación del profesorado: no tiene sentido recomendar insistentemente a los profesores de primaria y secundaria la introducción de orientaciones basadas en la indagación y dejar que la universidad siga practicando el chalk and talk con los futuros profesores. 
¿Cómo extrañarse de que, como afirma el Informe Rocard, mientras la comunidad de investigadores en educación científica está de acuerdo en que las prácticas educativas basadas en la indagación son más efectivas, en la mayor parte de los países europeos la enseñanza real no siga esta orientación? No es únicamente dando a conocer los resultados de la investigación y promoviendo redes de contacto entre docentes, para difundir ejemplos de aplicación de las nuevas estrategias, como se conseguirá implicarlos en la necesaria renovación de la enseñanza.

Esto es algo que ya había sido señalado por diversos autores (Briscoe, 1991; Gil et ál., 1991; Maiztegui et ál., 2000): es necesario que los profesores participemos en la construcción de los nuevos conocimientos didácticos, abordando los problemas que la enseñanza nos plantea. Sin esa participación, no sólo resulta difícil que los profesores y profesoras hagan suyos y lleven eficazmente adelante los cambios curriculares, sino que cabe esperar una actitud de rechazo que se apoya en problemas organizativos y sindicales o en preconcepciones docentes, como la dificultad de trabajar con la creciente diversidad de los jóvenes que prolongan sus estudios.

Nos encontramos, pues, con un doble reduccionismo que es preciso superar: limitar la formación del profesorado a los niveles de primaria y secundaria y reducir dicha formación a transmitir a los docentes los conocimientos necesarios, lo que supone una grave incoherencia entre la formación que se proporciona y lo que se pretende que hagan en el aula.
Por otra parte, ¿cuáles son esos "conocimientos necesarios" que los profesores debieran adquirir? En el Informe Rocard (2007) tan sólo se hace referencia a dos elementos: los contenidos científicos de las asignaturas impartidas y las nuevas propuestas de aprendizaje por indagación, concebidas para favorecer el aprendizaje y, muy en particular, el interés de los estudiantes por las ciencias. Nada se dice, sin embargo, acerca de los contenidos científicos, lo que deja suponer que se consideran adecuados los que se adquieren en una facultad de ciencias. Tropezamos así con un nuevo y serio reduccionismo.

En efecto, existe un acuerdo general en que el conocimiento profundo de la materia que se ha de impartir constituye un requisito imprescindible para una enseñanza de calidad. La investigación ha mostrado que la falta de dicho conocimiento dificulta muy seriamente que los profesores afectados puedan valorar correctamente los resultados de la enseñanza o participar en la elaboración de propuestas innovadoras (Tobin y Espinet, 1989). Pero es preciso ser conscientes de que un buen conocimiento de la materia va más allá de lo que suele impartirse en las facultades de ciencias y no se reduce a conocer los hechos, leyes y teorías que conforman el cuerpo de conocimientos científicos. Un buen conocimiento de la materia para un docente, tanto de secundaria como de universidad, supone también, entre otros aspectos (Gil Pérez y Vilches, 2004):

- Conocer los problemas que originaron la construcción de dichos conocimientos y cómo llegaron a articularse en cuerpos coherentes, evitando así 
visiones estáticas y dogmáticas que deforman y empobrecen la naturaleza del conocimiento científico (Fernández et ál., 2002). Se trata, en definitiva, de conocer la Historia de las Ciencias, no sólo como un aspecto básico de la cultura científica general, sino, primordialmente, como una forma de asociar los conocimientos científicos con los problemas que originaron su construcción, dentro de un contexto político, económico, cultural y social específico (Bell y Lederman, 2003; Garay, Gallego y Pérez Miranda, 2006), sin lo cual dichos conocimientos aparecen como construcciones arbitrarias (Otero, 1989; Matthews, 1994). Se puede así, además, conocer cuáles fueron las dificultades, los obstáculos epistemológicos que hubo que superar, lo que constituye una ayuda imprescindible para comprender las dificultades de los estudiantes.

- Conocer las estrategias empleadas en la construcción de los conocimientos, es decir, la forma en que los científicos y tecnólogos abordan los problemas, las características más notables de su actividad, los criterios de validación y aceptación de las teorías científicas. Se trata, como es lógico, de un conocimiento esencial para salir al paso de visiones deformadas y empobrecidas de las actividades científico-tecnológicas, socialmente aceptadas (Gallego Torres, 2002) y que la enseñanza suele reforzar por acción u omisión, contribuyendo al desinterés y rechazo de los estudiantes (Fernández et ál., 2002; Gil Pérez, Vilches et ál., 2005). Por otra parte, sin un conocimiento en profundidad de estas estrategias características del trabajo científico, resulta imposible, obviamente, orientar adecuadamente el aprendizaje de los estudiantes como una actividad de indagación.

- Conocer, en particular, las interacciones Ciencia, Tecnología, Sociedad y Ambiente, CTSA, que impregnan la construcción de conocimientos, sin ignorar el carácter a menudo conflictivo del papel social de las ciencias y la necesidad de la toma de decisiones en torno a los graves problemas que afectan a la Humanidad. En efecto, el trabajo de los hombres y mujeres de ciencia, como cualquier otra actividad humana, no tiene lugar al margen de la sociedad en que viven y se ve afectado, lógicamente, por los problemas y circunstancias del momento histórico, del mismo modo que su acción tiene una clara influencia sobre el medio físico y social en que se inserta. Señalar esto puede parecer superfluo; sin embargo, la idea de que hacer Ciencia es poco menos que encerrarse en una torre de marfil, desconectado de la realidad, constituye una imagen tópica muy extendida y a la que nuestra enseñanza lamentablemente contribuye con su reducción a la transmisión de contenidos conceptuales y, a lo sumo, entrenamiento en alguna destreza, pero dejando de lado los aspectos históricos, sociales, que enmarcan el desarrollo científico (Solbes y Vilches, 1997; Fernández et ál., 2002).

Un buen conocimiento de la materia supone, además, tener algún conocimiento de los desarrollos científicos recientes, sus implicaciones y sus perspectivas, para poder transmitir una visión dinámica, no cerrada, de la 
Ciencia, así como adquirir conocimientos de otras disciplinas relacionadas, para abordar problemas "puente", las interacciones entre distintos campos y los procesos de unificación que constituyen momentos cumbre del desarrollo científico.

Por otra parte, las necesidades formativas de los profesores de materias científicas no se limitan a este conocimiento en profundidad de la disciplina, sino que incluyen muchos otros conocimientos específicos de la docencia cuando ésta no se limita a la mera transmisión de conocimientos: se precisa, entre otras cosas:

Saber seleccionar contenidos que den una visión adecuada de las materias que se pretenden enseñar y sean asequibles a los alumnos y capaces de despertar su interés.

Saber diseñar programas adecuados de actividades para orientar la indagación de los estudiantes, promoviendo su inmersión en la cultura científica.

Facilitar el funcionamiento de equipos de trabajo y los intercambios enriquecedores entre equipos.

Dirigir y evaluar la actividad de los estudiantes, concibiendo la evaluación como un instrumento de regulación y mejora continuada del proceso de enseñanza y de aprendizaje, que pueda ser percibida por los estudiantes como una ayuda real, generadora de expectativas positivas y que contribuya a un buen clima de funcionamiento del aula, promoviendo actuaciones en el campo de la educación en valores propios de una ciudadanía activa y democrática, etc.

En particular, queremos resaltar el papel esencial que representan (o, mejor dicho, habrían de representar) en la for- mación del profesorado el conocimiento y análisis crítico del pensamiento docente espontáneo, fruto de una prolongada impregnación ambiental en prácticas que conciben la enseñanza como actividad de mera transmisión, sin apenas exigencias. Dicho pensamiento espontáneo constituye a menudo un serio obstáculo a la renovación de la enseñanza (Porlán, 1993; Bell, 1998; Gil Pérez y Vilches, 2004) y la familiarización con el cuerpo de conocimientos teóricos que la investigación e innovación han ido construyendo en torno a los problemas que plantea el proceso de enseñanza/aprendizaje de las ciencias (Gabel, 1994; Fraser y Tobin, 1998; Perales y Cañal, 2000).

La formación del profesorado y la propia actividad docente han dejado, por tanto, de concebirse como tareas sencillas, cuya preparación y puesta en práctica requieren escasa dedicación y esfuerzo. Dicha formación se revela, por el contrario, como una tarea compleja que exige romper con concepciones simplistas, superando reduccionismos como los analizados, y que ha de extenderse necesariamente al nivel universitario. La necesidad de esta ruptura con concepciones simplistas se ve reforzada cuando se toman en consideración las nuevas tareas que la sociedad exige hoy a la educación científica. Abordaremos esta cuestión en el siguiente apartado.

\section{Nuevas necesidades formativas ¿más dificultades 0 avance hacia una educación científica de calidad?}

A menudo se asocian las dificultades a las que se enfrenta hoy la educación científica, en particular en el nivel secundario, al profundo cambio que se ha producido en los objetivos asignados a la 
misma al apostarse por la alfabetización científica del conjunto de la ciudadanía, siendo así que el profesorado había sido preparado para seleccionar y formar una minoría de futuros científicos.

No vamos aquí a justificar la necesidad de la alfabetización científica del conjunto de ciudadanas y ciudadanos. Nos remitiremos a la abundante literatura que ha fundamentado dicha necesidad (Bybee y DeBoer, 1994; Bybee, 1997; Fourez, 1997; DeBoer, 2000; Marco, 2000; Gil Pérez y Vilches, 2001 y 2005). Como expresión del apoyo social que concita la alfabetización científica podemos mencionar la Declaración de la Conferencia Mundial sobre la Ciencia para el siglo XXI, auspiciada por la Unesco y el Consejo Internacional para la Ciencia (Budapest, 1999) ${ }^{4}$ en la que se afirma que hoy más que nunca es necesario fomentar y difundir la alfabetización científica en todas las culturas y en todos los sectores de la sociedad, a fin de mejorar la participación de los ciudadanos en la adopción de decisiones relativas a la aplicaciones de los nuevos conocimientos.

Nuestro propósito aquí es dilucidar qué nuevas tareas y orientaciones se asignan hoy día a la educación científica de la ciudadanía y, en particular, en qué medida este objetivo de alfabetización científica del conjunto de la ciudadanía entra en conflicto con el de la preparación de futuros científicos. Un conflicto que contribuiría, en opinión de algunos, a las actuales dificultades de la enseñanza de las ciencias, afectan-

4 http://www.unesco.org/science/wcs/esp/ declaracion_s.htm do particularmente a los docentes de secundaria. En efecto, una tesis comúnmente aceptada por los diseñadores de currículos y los profesores de ciencias es que la educación científica ha estado orientada hasta aquí para preparar a los estudiantes como si todos pretendieran llegar a ser especialistas en Biología, Física o Química. Por ello -se afirma- los currículos planteaban, como objetivos prioritarios, que los estudiantes supieran, fundamentalmente, los conceptos, principios y leyes de esas disciplinas. Dicha orientación ha debido modificarse -se explica- a causa de que la educación científica se plantea ahora como parte de una educación general para todos los futuros ciudadanos y ciudadanas.

Algunos investigadores parecen aceptar esta contraposición entre educación científica para todos y preparación de los futuros científicos. Así, Bybee (1997) sostiene que, en muchos aspectos, los objetivos de enseñar ciencias para futuros científicos y para futuros ciudadanos son contradictorios, no complementarios. Un programa de ciencias para futuros científicos contemplaría la Ciencia "desde dentro", mientras que el programa para futuros ciudadanos lo haría desde la perspectiva de la sociedad. Ello es lo que justifica, se afirma, el énfasis actual de los currículos en los aspectos sociales y personales (National Academy of Science, 1995), puesto que se trata de ayudar a la gran mayoría de la población a tomar conciencia de las complejas relaciones Ciencia - Sociedad, para permitirles participar en la toma de decisiones y, en definitiva, a considerar la Ciencia como parte de la cultura de nuestro tiempo. Dichos cambios en la orientación de la enseñanza 
secundaria se verían dificultados por el hecho de que el profesorado habría sido formado para preparar a futuros científicos. Más aún, esta apuesta por una educación científica orientada a la formación ciudadana, en vez de la preparación de futuros científicos, genera resistencias en numerosos profesores, quienes argumentan, legítimamente, que la sociedad necesita científicos y tecnólogos que han de formarse y ser adecuadamente seleccionados desde los primeros estadios.

Es preciso pronunciarse con claridad contra estas explicaciones de los cambios curriculares y de las dificultades actuales de la educación científica. Hoy sabemos que una educación científica, como la practicada hasta aquí, centrada en los aspectos conceptuales es igualmente criticable como preparación de futuros científicos. En efecto, esta orientación, en primer lugar, transmite una visión deformada y empobrecida de la actividad científica, que no sólo contribuye a una imagen pública de la Ciencia como algo ajeno e inasequible -cuando no directamente rechazable-, sino que puede hacer disminuir el interés de los jóvenes por dedicarse a la misma. Ya nos hemos referido a la gravedad y extensión de estas deformaciones, puestas de relieve por numerosas investigaciones (Fernández et ál., 2002; Gallego Torres, 2002).

Dichas investigaciones han mostrado las discrepancias entre la visión de la Ciencia proporcionada por la Epistemología contemporánea y ciertas concepciones y prácticas docentes, ampliamente extendidas, que conciben la actividad científica como un conjunto rígido de etapas a seguir mecánicamente (ob- servación, acumulación de datos, etc.), resaltando lo que supone tratamiento cuantitativo, control riguroso, etc., pero olvidando -o, incluso, rechazando- todo lo que significa invención, creatividad, duda. Por otra parte, los conocimientos científicos fruto de esta metodología, aparecen como "descubrimientos" de genios aislados, encerrados en torres de marfil y ajenos a las necesarias tomas de decisión, ignorándose así tanto el papel del trabajo colectivo, de los intercambios entre equipos, como las complejas relaciones entre Ciencia, Tecnología y Sociedad, CTS. Todo ello hace que una enseñanza supuestamente dirigida a la formación de científicos, proporcione una imagen de la Ciencia que, además de falsa, resulta escasamente atractiva, dificultando la génesis de vocaciones científicas (Solbes y Vilches, 1997).

Cabe resaltar, además, que esta enseñanza centrada en los aspectos conceptuales, dificulta, paradójicamente, el aprendizaje conceptual (amén de proporcionar una visión empobrecida de la Ciencia). En efecto, la investigación didáctica, tanto en el campo de las preconcepciones como en el de los trabajos prácticos, la resolución de problemas, etc., está mostrando que "los estudiantes desarrollan mejor su comprensión conceptual y aprenden más acerca de la naturaleza de la Ciencia cuando participan en investigaciones científicas, con tal que haya suficientes oportunidades y apoyo para la reflexión" (Hodson, 1992). Dicho con otras palabras, lo que la investigación está mostrando es que la comprensión significativa de los conceptos exige superar el reduccionismo conceptual y plantear la enseñanza de las ciencias como una actividad, próxima a 
la investigación científica, que integra los aspectos conceptuales, procedimentales y axiológicos.

Tras la idea de alfabetización científica no debe verse, pues, una "desviación" o "rebaja" para hacer accesible la Ciencia a la generalidad de los ciudadanos, sino una reorientación de la enseñanza absolutamente necesaria también para los futuros científicos; necesaria para modificar la imagen deformada de la Ciencia socialmente aceptada y luchar contra los movimientos anti-ciencia que se derivan; necesaria incluso, insistimos, para hacer posible una adquisición significativa de los conceptos. Y aunque es cierto que, como afirma Bybee (1997), la alfabetización científica debe concebirse como un continuo que puede desarrollarse a lo largo de la vida de cada cual (y que, por supuesto, unos desarrollarán más que otros), resulta esencial reconocer que la mejor formación científica inicial que puede recibir un futuro científico coincide con la orientación a dar a la alfabetización científica del conjunto de la ciudadanía (Gil Pérez y Vilches, 2005). La reorientación de la enseñanza que ello exige no sólo no introduce nuevas dificultades, sino que abre perspectivas de solución, al convertir la inmersión en la cultura científica en una tarea abierta y creativa para los estudiantes y para el propio profesorado.

Cabe señalar que la necesidad de esta nueva orientación de la educación científica, avalada también por el propio Informe Rocard, se ha visto reforzada por los llamamientos de diversos organismos y conferencias internacionales para que los educadores contribuyamos a que los ciudadanos adquieran una correcta percepción de los problemas y desafíos estrechamente interconectados a los que se enfrenta hoy la humanidad (Vilches y Gil Pérez, 2003), que configura lo que Bybee (1991) ha denominado acertadamente una situación de emergencia planetaria, y puedan así participar en la necesaria toma de decisiones fundamentadas. Aunque existen antecedentes importantes, como la Conferencia Internacional sobre el Medio Ambiente Humano celebrada en Estocolmo en 1972, este llamamiento adquirió una gran relevancia en 1992, durante la Cumbre de la Tierra convocada por la Organización de las Naciones Unidas en Río de Janeiro. Con ello se pretendía hacer posible la participación ciudadana en la toma fundamentada de decisiones, así como los necesarios cambios de comportamiento para evitar que las condiciones de vida de la especie humana lleguen a degradarse de manera irreversible. En dicha Conferencia, en la que participaron los gobiernos de la mayoría de países de la Tierra y miles de Organizaciones no Gubernamentales, se pidió explícitamente a través de su Agenda 21 (Naciones Unidas, 1992) que todos los educadores, cualquiera sea nuestro campo específico de trabajo, contribuyamos a hacer posible la participación ciudadana en la búsqueda de soluciones. Estos llamamientos han culminado con la institución por parte de Naciones Unidas de una Década de la Educación por un futuro sostenible, 2005-2014, que busca lograr la implicación de todos los educadores 5 .

Pero los llamamientos para hacer frente a la situación de emergencia pla-

\footnotetext{
5 http://www.oei.es/decada
} 
netaria no se limitan a los educadores. La propia comunidad científica ha sido objeto de similares peticiones. Podemos mencionar, por ejemplo, el llamamiento realizado a fines del siglo XX por Jane Lubchenco, presidenta de la American Association for the Advancement of Science (la más importante asociación científica a nivel mundial, tanto por el número de miembros como por la cantidad de premios Nobel y científicos de alto nivel que forman parte de la misma, y editora de la prestigiosa revista Science). Lubchenco reclamaba que el siglo XXI fuera, para la Ciencia, el siglo del medio ambiente y que la comunidad científica "reorientara su maquinaria" hacia la resolución de los problemas que amenazan el futuro de la humanidad.

Los profesores y profesoras de ciencias y los investigadores en el campo de la educación científica debemos responder a este doble llamamiento e incorporar la problemática de la sostenibilidad a nuestro trabajo. En la página web http://www.oei.es/decada se recogen numerosos ejemplos de acciones, porque las posibilidades de contribución a los objetivos de la Década de la Educación por un futuro sostenible son múltiples $\mathrm{y}$, a menudo, pueden realizarse como parte de nuestro trabajo habitual, que queda así enriquecido. Contribuir al llamamiento de las Naciones Unidas puede verse, además de como un compromiso ineludible, como una ocasión de incrementar la relevancia de nuestras investigaciones y el interés de nuestra acción educativa. Un objetivo para el que constituirá un gran impulso la incorporación de la problemática de la sostenibilidad en los currículos de la educación básica, como ya viene haciéndose en numerosos países.

Todo apunta, pues, al enriquecimiento de la educación científica, al aprendizaje como investigación o indagación, es decir, como participación en la re-construcción de conocimientos, partiendo de problemas relevantes que pongan en juego las estrategias creativas de la actividad científica y las relaciones Ciencia-Tecnología-Sociedad-Ambiente que la enmarcan. Un enriquecimiento que aparece, además, como el camino para romper con el desinterés por la cultura científica. La formación de los docentes ha de permitir esta orientación del aprendizaje como impregnación en la cultura científica o "enculturación científica” (Pessoa de Carvalho, 2006). Y ello exige que su propia formación sea coherente con dicha impregnación. Nos referiremos ahora a las estrategias necesarias para ello.

\section{Nuevas estrategias para la formación del profesorado de ciencias}

Como ya hemos señalado, la formación de un profesorado al que se pide que oriente el trabajo en el aula como una indagación que permita a los estudiantes participar en la construcción de los conocimientos (Rocard et ál., 2007), no puede consistir en la transmisión de conocimientos científicos presentados de forma descontextualizada, puramente operativa, completados con propuestas educativas elaboradas por expertos y presentadas para su aplicación. ¿Cómo orientar, pues, dicha formación?

De acuerdo con lo mostrado por la investigación acerca de los procesos de aprendizaje, la estrategia que parece potencialmente más fructífera para la 
formación del profesorado consistiría en:

- Favorecer que los futuros docentes aprendan los contenidos de su materia mediante un proceso de investigación e impregnación en la cultura científica, como el que se pretende que utilicen después con sus alumnos y alumnas. Ello exige que la enseñanza universitaria modifique radicalmente su orientación (Martínez Torregrosa, Gil Pérez y Martínez Sebastián, 2003), algo que, lamentablemente, no tiene visos de producirse en lo inmediato: el Informe Rocard, por ejemplo, no hace mención alguna a la universidad. Por ello, en tanto esta transformación fundamental no tenga lugar, serán precisos cursos y talleres complementarios que permitan vivenciar esta forma de orientar el aprendizaje de las ciencias.

- Orientar su formación didáctica, es decir su apropiación del cuerpo de conocimientos elaborado por los investigadores e innovadores en didáctica de las ciencias, también como un proceso de indagación, implicando al profesorado en la investigación de los problemas de enseñanza/aprendizaje de las ciencias que les plantea su actividad docente (Gil Pérez, Furió y Gavidia, 1998; Gil Pérez y Vilches, 2004).

No se trata, claro está, de que cada profesor o grupo de profesores tenga que construir aisladamente, por sí mismo, todos los conocimientos elaborados por la comunidad científica, sino de proporcionarle la ayuda necesaria para que participe en la reconstrucción y apropiación de dichos conocimientos.
Esta propuesta formativa puede describirse, más bien, con la metáfora de "los investigadores noveles" que, como es bien conocido, son capaces de realizar notables progresos en la medida en que investigan contando con el apoyo de investigadores expertos, es decir, de investigadores que conocen bien la problemática que han de investigar los noveles y que pueden darles la retroalimentación adecuada ( $\sin$ recurrir a una inefectiva transmisión de los conocimientos, al contrario, permitiéndoles replicar trabajos cuyos resultados, en buena medida, ya conocen los expertos).

Esta orientación de la formación del profesorado exige contar con un número creciente de profesores capaces de tutorar a otros colegas y de favorecer su implicación en el tratamiento de los problemas que el proceso de enseñanza/aprendizaje de las ciencias plantea, es decir, capaces de dirigir la actividad de grupos de investigadores noveles, al tiempo que ellos mismos siguen afianzando su formación y enriqueciendo su bagaje científico como miembros de una comunidad de investigadores/ innovadores en didáctica de las ciencias, en la que se apoyan y a cuyo cuerpo de conocimientos contribuyen a su vez. Más precisamente, dicha estrategia habría de poseer, entre otras, las siguientes características:

- Ser concebida en íntima conexión con la propia práctica docente, como tratamiento de los problemas de enseñanza/aprendizaje que dicha práctica plantea.

- Orientada a favorecer la vivencia de propuestas innovadoras y la reflexión didáctica explícita, cuestionando el pensamiento y comportamiento 
docente espontáneos, es decir, cuestionando el carácter natural de "lo que siempre se ha hecho".

- Diseñada para incorporar al profesorado a la investigación e innovación en didáctica de las ciencias y, de este modo, favorecer su familiarización con el cuerpo de conocimientos específico de la didáctica de las ciencias e incorporar a la comunidad científica en este campo.

Esta estrategia de autoformación colectiva constituye, hemos de reconocer, una propuesta particularmente exigente y difícil de implementar. Lo ideal sería que existiera ya una tradición de trabajo colectivo en el profesorado, con equipos capaces de incorporar a las nuevas generaciones de docentes y de facilitarles, a través del trabajo común, la formación necesaria (como ocurre en cualquier tarea con aspiración científica). Pero es obvio que hoy apenas existen tales equipos y que no pueden improvisarse ni constituirse "por decreto", cuando falta la tradición del trabajo científico (Porlán, 1998). Por otra parte, las condiciones laborales vigentes del profesorado no tienen en cuenta esta necesidad de trabajo colectivo como parte esencial de la tarea docente: los horarios lectivos recargados, por ejemplo, constituyen una dificultad muy seria. Por ello, el establecimiento de una estructura plenamente efectiva de formación continuada del profesorado ha de contemplarse como un objetivo a largo plazo, lo que no significa, ni mucho menos, posponer las acciones necesarias para lograrlo. Y por la misma razón no puede esperarse, a corto plazo, la plena efectividad de unas reformas educativas, cuya necesidad nadie discute hoy.
Lo que se puede hacer ahora distará bastante, inevitablemente, de esta estrategia ideal de autoformación colectiva y de participación del profesorado en la construcción de las nuevas orientaciones curriculares. Es preciso ser conscientes de ello, pero también es preciso tener claro cuál es el objetivo y plantear las acciones posibles en esa perspectiva de creación de equipos autónomos, integrados en la comunidad de investigadores e innovadores en didáctica de las ciencias.

Una cuestión clave, pues, es ver qué se puede hacer hoy y cómo optimizar los recursos disponibles para avanzar en la perspectiva correcta. En ese sentido, son posibles muchas acciones, siempre que se planteen, insistimos, en una perspectiva de implicación de los profesores en el tratamiento de los problemas de enseñanza/aprendizaje de las ciencias relacionados con su actividad docente. No podemos pasar aquí a analizar -ni siquiera a presentar resumidamente- estas distintas iniciativas posibles, pero creemos que vale la pena referirse brevemente a una de las acciones que consideramos potencialmente más fructíferas para optimizar los recursos humanos disponibles en nuestros países: la constitución de una comunidad de Formadores de equipos docentes que incorpore a aquellos profesores que, por su labor innovadora, sus investigaciones, etc., puedan realizar aportes valiosos en la formación continuada de sus colegas y, fundamentalmente, servir de dinamizadores en la fase inicial. Ello es coherente con lo que propone el Informe Rocard (2007) en su segunda recomendación acerca del desarrollo de redes de profesores, aunque, en nuestra 
opinión, habrá que hacer énfasis en que se precisan redes de equipos y no la simple puesta en común, a través de la red, de trabajos individuales.

El punto de partida de esta propuesta es el reconocimiento de que, independientemente de los impulsos oficiales, en todos los países se producen iniciativas de autoformación, de implicación en tareas innovadoras, etc., con las que una serie de profesores adquieren una preparación y, sobre todo, una disposición para la renovación de la enseñanza que puede ser de gran utilidad en un plan de formación continuada del profesorado.

Se trataría, pues, en una primera fase, de organizar talleres para aquellos profesores que disponen ya de una base, con el objeto de proporcionarles la ocasión de un trabajo colectivo de cierta profundidad en torno a la renovación de la enseñanza y en torno a la formación continuada del profesorado. Estos talleres habrían de diseñarse muy cuidadosamente, con un claro hilo conductor y con la duración suficiente para permitir a los asistentes:

- Apropiarse colectivamente de los aportes de la innovación e investigación en didáctica de la propia área,

- Poner en común sus experiencias y

- Llegar a conformar una comunidad cohesionada, capaz de cooperar eficazmente en los procesos de renovación curricular y en las tareas de formación continuada del profesorado.

Esta propuesta se inspira en las experiencias de formación de formadores, llevadas a cabo en algunos países con resultados, en general, positivos, tanto en lo que se refiere a la preparación adquirida por los asistentes a dichos cursos como, sobre todo, al papel que han desempeñado después en los programas de formación permanente del profesorado. Es preciso llamar la atención, sin embargo, contra una interpretación de este proyecto como una simple transmisión de propuestas "en cascada". Insistimos en que el objetivo es potenciar la formación de equipos docentes interconectados, es decir, que formen parte de una auténtica comunidad científica, capaces de abordar colectivamente y con continuidad los problemas que plantea el proceso de enseñanza/aprendizaje de su área.

Ésta es, en resumen, la idea esencial de la propuesta que la investigación realizada hasta aquí parece apoyar: comenzar potenciando a los profesores que posean ya una cierta preparación y, sobre todo, una buena disposición, poniéndolos en situación de apropiarse activamente-como investigadores noveles- del cuerpo de conocimientos disponible en el campo de la Didáctica de las Ciencias, o del área de que se trate, preparándolos a tiempo para desempeñar el papel de impulsores y orientadores de futuros investigadores noveles.

\section{Conclusión y perspectivas}

El conjunto de saberes y destrezas al que nos hemos ido refiriendo proporciona una visión rica y compleja de la actividad docente que reclama, como hemos visto, una investigación e innovación permanente. Ello debe ser resaltado, una vez más, como un resultado positivo, por cuanto convierte la docencia en una tarea abierta y creativa. Pero es preciso reconocer que puede generar también cierto malestar entre los profesores, quienes a menudo se preguntan si tiene sentido esperar que un profesor llegue 
a poseer tantos conocimientos como los que se han contemplado como necesarios para una docencia de calidad.

La respuesta ha de ser clara y contundente: por supuesto que ello es imposible, pero es la propia cuestión la que carece de sentido. En efecto, cualquier estudio sobre Epistemología de la Ciencia revela unas exigencias para el trabajo científico al menos tan amplias como las señaladas para el trabajo docente; pero a ningún científico se le exige que posea el conjunto de saberes y destrezas necesarios para el desarrollo científico: se tiene muy claro que se trata de una tarea colectiva. Del mismo modo, el trabajo docente tampoco es, o mejor dicho, no debería ser, una tarea aislada, y ningún profesor ha de sentirse desbordado por un conjunto de saberes que, con toda seguridad, sobrepasan las posibilidades de un ser humano. Lo esencial es que pueda darse un trabajo colectivo en todo el proceso de enseñanza/aprendizaje: desde la preparación de las clases hasta la evaluación.

\section{Referencias}

Abd-El-Khalick, F., Boujaoude, S., Duschl, R., Lederman, N.G., Mamlok-Naaman, R., Hofstein, A., Niaz, M., Treagust, D. y Tuan, H. (2004). Inquiry in Science Education: International perspectives, Science Education, 88 (3), 397-419.

Anderson, R.D. y Mitchener, C.P. (1994). Research on science teacher education. En Gabel, D. L. (Ed.). Handbook of Research on Science Teaching Education. New York: Macmillan Pub. Co.

Ausubel D. P. (1978). Psicología Educativa. Un punto de vista cognoscitivo. México: Trillas
Con este punto de vista, la complejidad de la actividad docente deja de verse como un obstáculo a la eficacia y un factor de desánimo para convertirse en una invitación a romper con la inercia de una enseñanza monótona y sin perspectivas, y a aprovechar la enorme creatividad potencial de la enseñanza. Se trata, en definitiva, de orientar dicha tarea docente como un trabajo colectivo de innovación, investigación y formación permanente.

Reconocemos, para terminar, que se trata de una propuesta muy exigente desde muchos puntos de vista, incluido el presupuestario, puesto que la incorporación a tareas de investigación e innovación es incompatible con horarios lectivos desmesurados. Se precisa, por tanto, una profunda reconsideración del papel de la Educación en nuestras sociedades. No basta con atribuir a la Educación -y, en particular, a la educación científica- un papel esencial en el desarrollo de los pueblos. Hay que hacerlo posible. $\boldsymbol{\Delta}$

Bell, B. (1998). Teacher development in science education. En Fraser, B. J. y Tobin, K. G. (Eds). International Handbook of Science Education, Dordrecht: Kluber.

Bell, R. y Lederman, N. (2003). Understandings of the nature of Science and decision making on Science and Technology based Issues. Science Education, 87 (3), 352-377.

Black, P. (2000). Physics Education in the New Millenium. En Black, P., Drake, G. y Jossem, L. (Eds.). GIREP 2000. Physics as it Enter a New Millenium, 80-85. 
Briscoe, C. (1991). The dynamic interactions among beliefs, role metaphors and teaching practices. A case study of teacher change. Science Education, 75 (2), 185-199.

Bybee, R. (1991). Planet Earth in Crisis: How Should Science Educators Respond? The American Biology Teacher, 53(3), 146-153.

Bybee, R. (1997). Towards an Understanding of Scientific Literacy. En Gräber, W. and Bolte, C. (Eds.) Scientific Literacy. Kiel: IPN.

Bybee, R. y DeBoer, G.E. (1994). Research on goals for the science curriculum. En Gabel, D.L. Handbook of Research en Science Teaching and Learning. New York: McMillan P.C.

DeBoer, G. E. (2000). Scientific literacy: another look at its historical and contemporary meanings and its relationship to science education reform. Journal of Research in Science Teaching, 37(6), 582-601.

Fernández, I., Gil-Pérez, D., Carrascosa, J., Cachapuz, J. y Praia, J. (2002). Visiones deformadas de la ciencia transmitidas por la enseñanza. Enseñanza de las Ciencias, 20 (3), 477-488.

Fourez, G. (1997). Alfabetización científica y tecnológica. Acerca de las finalidades de la enseñanza de las ciencias. Colihue: Buenos Aires.

Fraser, B. y Tobin, K. G. (Eds.) (1998). International Handbook of Science Education London: Kluwer Academic Publishers.

Gabel, D. L (Ed). (1994). Handbook of Research on Science Teaching and Learning. New York: MacMillan Pub. Co.

Gallego Torres, A. P. (2002). Contribución del cómic a la imagen de la ciencia. Tesis Doctoral. Valencia: Universidad de Valencia.
Garay, F., Gallego, R. y Pérez Miranda, R. (2006). Un análisis históricoepistemológico de los trabajos de Mendeleiev sobre la periodicidad química. Tecné, Episteme y Didaxis, 20, 110-123.

Gil-Pérez, D. (1991). ¿Qué han de saber y saber hacer los profesores de ciencias? Enseñanza de las Ciencias, 9 (1), 69-77.

Gil, D., Carrascosa, J. Furió, C. y MartínezTorregrosa, J. (1991). La enseñanza de las ciencias en la educación secundaria. Barcelona: Horsori.

Gil, D., Furió, C. y Gavidia, V. (1998). El profesorado y la reforma educativa en España. Investigación en la Escuela, 36, 49-64.

Gil- Pérez, D., Macedo, B., Martínez Torregrosa, J., Sifredo, C., Valdés, P. \& Vilches, A. (Eds.). (2005).¿Cómo promover el interés por la cultura científica? Una propuesta didáctica fundamentada para la educación científica de jóvenes de 15 a 18 años. Santiago: OREALC/UNESCO. (Todo el contenido del libro accesible en http://www.oei.es/decada/libro. htm).

Gil-Pérez, D. y Vilches, A. (2001). Una alfabetización científica para el siglo XXI. Obstáculos y propuestas de actuación. Investigación en la Escuela, $43,27-37$.

Gil-Pérez, D. y Vilches, A. (2004). La formación del profesorado de ciencias de secundaria y de universidad. La necesaria superación de algunos mitos bloqueadores”. Educación Química, Vol. 15 (1), 43-51.

Gil-Pérez, D. y Vilches, A. (2005). Contribution of Science and Technological Education to Citizens' Culture. Canadian Journal of Science, Mathematics 
and Technology Education, 5(2), 2005, 253-263.

Gil-Pérez, D., Vilches, A., Fernández, I., Cachapuz, A., Praia, J., Valdés, P. \& Salinas, J. (2005). Technology as 'Applied Science': a Serious misconception that Reinforces Distorted and Impoverished Views of Science. Science \& Education, 14, 309-320.

Hewson, P. W. y Hewson, M. G. (1988). On appropriate conception of teaching science: a view from studies of science learning. Science Education, 72 (5), 597-614.

Hodson, D. (1992). In search of a meaningful relationship: an exploration of some issues relating to integration in science and science education. International Journal of Science Education, 14(5), 541-566.

Lee, O., Buxton, C., Lewis, S. y LeRoy, K. (2006). Science inquiry and student diversity: Enhanced abilities and continuing difficulties after an instructional intervention. Journal of Research in Science Teaching, 43 (7), 607-656.

McDermott, L. C. (1990). A perspective on teacher preparation in Physics and other sciences: The need for special science courses for teachers", American Journal of Physics, 58 (8), 734-742.

Maiztegui, A., González, E., Tricárico, H. R., Salinas, J., Pessoa De Carvalho, A. y Gil Pérez, D. (2000). "La formación de los profesores de ciencias en Iberoamérica". Revista Iberoamericana de Educación, 24, 163-187.

Marco, B. (2000). La alfabetización científica. En Perales, F. y Cañal, P. (Eds): Didáctica de las Ciencias Experimentales, 141-164. Alcoi: Marfil.
Martínez Torregrosa, J., Gil Pérez, D. \& Martínez Sebastián, B. (2003). La universidad como nivel privilegiado para un aprendizaje como investigación orientada. En Monero, C. y Pozo, J. I. La Universidad ante la nueva cultura educativa, Barcelona: Editorial Síntesis, 237-260.

Matthews, M. R. (1994). Historia, filosofía y enseñanza de las ciencias: la aproximación actual. Enseñanza de las Ciencias, 12 (2), 255-277.

Mumby, H. y Russell, T. (1998). Epistemology and context in research on learning to teach Science. En Fraser, B. J. y Tobin, K. G (Eds.). International Handbook of Science Education. Dordrecht: Kluber.

Organización de las Naciones Unidas (1992). UN Conference on Environment and Development, Agenda 21 Rio Declaration, Forest Principles. Paris: UNESCO.

National Academy of Sciences. (1995). National Science Education Standards. Washington, DC: National Academy Press.

Otero, J. (1989). La producción y la comprensión de la Ciencia: la elaboración en el aprendizaje de la ciencia escolar. Enseñanza de las Ciencias, 7 (3), 223-228.

Perales, J. y Cañal, P. (2000). Didáctica de las Ciencias: Teoría y Práctica de la Enseñanza de las Ciencias. Alcoi: Marfil.

Pessoa De Calvalho, A.M. (2006). Enseñar ciencias y, a la vez, promover la enculturación científica. Tecné, Episteme y Didaxis, 20, 3-4.

Porlán, R. (1993). Constructivismo y Escuela. Hacia un modelo de enseñanza- aprendizaje basado en la investigación. Sevilla: Diada. 
Porlán, R. (1998). Pasado, presente y futuro de la didáctica de las ciencias. Enseñanza de las Ciencias, 16 (1), 175-185.

Rocard, M., Csermely, P., Jorde, D., Lenzen, D., Walwerg-Henriksson, H. y Hemmo, V. (2007). Science Education Now: A Renewed Pedagogy for the Future of Europe. European Commission. Community Research. (En línea : http://ec.europa.eu/research/ science-society/document_library/ pdf_06/report-rocard-on-scienceeducation_en.pdf).

Rudolph, J. L. (2005). Inquiry, Instrumentality, and the Public Understanding of Science, Science Education, 89 (5), 803-821.

Solbes, J. y Vilches, A. (1997). STS interactions and the teaching of Physics and Chemistry. Science Education, 81(4), 377-386.
Tobin, K. y Espinet, M. (1989). Impediments to change: applications of coaching in high school science teaching. Journal of Research in Science Teaching, 26 (2), 105-120.

Trumbull, D. J., Grudens-Schuck, N. y

Bonney, R. (2005). "Developing materials to promote inquiry: lessons learned". Science Education, 89(6), 879-900.

Vilches, A. y Gil-Pérez, D. (2003). Construyamos un futuro sostenible. Diálogos de supervivencia. Madrid: Cambridge University Press.

Windschitl, M. (2005). The future of science teacher preparation in America: Where is the evidence to inform program design and guide responsible policy decisions? Science Education, 89 (4), 525-534. 